\title{
HOLOMORPHIC CURVES WITH MAXIMAL DEFICIENCY SUM
}

\author{
BY SEIKI MORI
}

Introduction. Let $f$ be a non-degenerate holomorphic mapping of the $m$ dimensional complex Euclidean space $\boldsymbol{C}^{m}$ into the $n$-dimensional complex projective space $\boldsymbol{P}^{n}$. Then, for any $q$ hyperplanes $\left\{H_{\nu}\right\}_{\nu=1}^{q} \subset \boldsymbol{P}^{n}$ in general position, the inequality so called Nevanlinna's defect relation $\sum_{\nu=1}^{q} \delta\left(H_{\nu}, f\right) \leqq n+1$ is well known (See Stoll [6] or Vitter [9]). What can we say about a mapping $f$ with maximal deficiency sum?

In the case $m=n=1$, the following result is known: If $f$ is a meromorphic function of finite order which satisfies $\delta(\infty, f)=1$ and $\sum_{a \neq \infty} \delta(a, f)=1$, then $f$ is of positive integral order and of regular growth (See Edrei-Fuchs [1]).

In the case when $m, n \geqq 1$, if there exist $n+1$ hyperplanes $\left\{H_{\nu}\right\}_{\nu=0}^{n} \subset \boldsymbol{P}^{n}$ in general position such that $\sum_{\nu=0}^{n} \delta\left(H_{\nu}, f\right)=n+1$, then $f$ is of positive integral order or infinite order and is of regular growth (Mori [4] or Noguchi [5]).

In this note we treat the case of holomorphic curves with maximal deficiency sum.

$\S 1$. Notations. Let $f: \boldsymbol{C} \rightarrow \boldsymbol{P}^{n}$ be a non-degenerate holomorphic curve and let $L$ be the standard line bandle over $\boldsymbol{P}^{n}$. For a homogeneous coordinate system $w=\left[w_{0}, \cdots, w_{n}\right]$ on $\boldsymbol{P}^{n}$,

$$
h_{\alpha}(w)=\sum_{k=0}^{n}\left|\frac{w_{k}}{w_{\alpha}}\right|^{2} \quad \text { in } \quad U_{\alpha}=\left\{w: w_{\alpha} \neq 0\right\}
$$

is a metric on $L \rightarrow \boldsymbol{P}^{n}$. Let $\phi=\left\{\phi_{\alpha}\right\} \in H^{0}\left(\boldsymbol{P}^{n}, O(L)\right)$ be a holomorphic section. Since $\left|\psi_{\alpha}(w)\right| / h_{\alpha}(w)=\left|\psi_{\beta}(w)\right| / h_{\beta}(w)$ on $U_{\alpha} \cap U_{\beta}$, we put

$$
|\psi|^{2}(w) \equiv \frac{\left|\psi_{\alpha}(w)\right|^{2}}{h_{\alpha}(w)}
$$

and call it the norm of $\phi$. We put $\omega=\omega_{L} \equiv \frac{\sqrt{-1}}{2 \pi} \partial \widehat{o} \log h_{\alpha}$ which is the curvature form on $L$. The quantity

Received October 10, 1977. 


$$
T(r, f) \equiv \int_{0}^{r} \frac{d t}{t} \int_{\mid z i<t} f^{*} \omega
$$

is called the characteristic function of $f$ where $f^{*} \omega$ denotes the pull back of the form $\omega$ by $f$. For a divisor $(\psi)$ of $\phi$, we denote by $n(t,(\psi))$ the number of points of $\left\{\left(f^{*} \psi\right) \cap(|z|<t)\right\}$ counting multiplicities, and put

$$
N(r,(\psi)) \equiv \int_{0}^{r} \frac{n(t,(\psi))}{t} d t
$$

For a hyperplane $H$ in $\boldsymbol{P}^{n}$, we choose a global holomorphic section $\phi \in H^{0}\left(\boldsymbol{P}^{n}\right.$, $O(L))$ such that $(\psi)=H$ and $|\psi| \leqq 1$, and put

$$
m(r, H) \equiv \frac{1}{2 \pi} \int_{0}^{2 \pi} \log \frac{1}{f^{*}|\phi|(z)} d \theta \quad\left(z=r e^{2 \rho}\right) .
$$

In particular, we use notations $T_{1}(r, g), m_{1}(r, \infty)=m_{1}(r, g)$ and $N_{1}(r, \infty)=N_{1}(r, g)$ for a meromorphic function $g: \boldsymbol{C} \rightarrow \boldsymbol{P}^{1}=\boldsymbol{C} \cup\{\infty\}$.

By Nevanlinna's first main theorem, we have

$$
T(r, f)=N(r, H)+m(r, H)+\log f^{*}|\psi|(0)
$$

provided that $f^{*} H \boxplus 0$. For a hyperplane $H \subset \boldsymbol{P}^{n}$, the quantity

$$
\delta(H, f) \equiv 1-\limsup _{r \rightarrow \infty} \frac{N(r, H)}{T(r, f)}\left(=\liminf _{r \rightarrow \infty} \frac{m(r, H)}{T(r, f)}\right)
$$

is called the deficiency of $H$. We define the order $\lambda$ and the lower order $\mu$ of $f$ as follows :

$$
\lambda=\limsup _{r \rightarrow \infty} \frac{\log T(r, f)}{\log r} \text { and } \quad \mu=\liminf _{r \rightarrow \infty} \frac{\log T(r, f)}{\log r} .
$$

§2. Statement of Theroem. Let $f: \boldsymbol{C} \rightarrow \boldsymbol{P}^{n}$ be a holomorphic curve and $w=\left[w_{0}, \cdots, w_{n}\right]$ a homogeneous coordinate system in $\boldsymbol{P}^{n}$. Then $f$ can be represented as $f=\left[f_{0}, \cdots, f_{n}\right]$, where $f_{3}$ are entire functions without common zeros. If $f=\left[g_{0}, \cdots, g_{n}\right]$ is another representation of $f$, then there is an entire function $\alpha(z)$ such that $g_{\jmath}(z)=e^{\alpha(z)} f_{j}(z)(\jmath=0, \cdots, n)$. We note that the characteristic function $T(r, f)$ can be written as

$$
T(r, f)=\frac{1}{4 \pi} \int_{0}^{2 \pi} \log \sum_{k=0}^{n}\left|f_{k}\left(r e^{i \theta}\right)\right|^{2} d \theta-\log \left(\sum_{k=0}^{n}\left|f_{k}(0)\right|^{2}\right)^{1 / 2}
$$

provided that $\sum_{k=0}^{n}\left|f_{k}(0)\right| \neq 0$.

THEOREM. Let $f \cdot \boldsymbol{C} \rightarrow \boldsymbol{P}^{n}$ be a non-degenerate holomorphic curve of finte order $\lambda$. If there are $q$ hyperplanes $\left\{H_{\nu}\right\}_{\nu=0}^{q-1} \subset \boldsymbol{P}^{n}(n+1 \leqq q \leqq+\infty)$ in general position such that $\lambda_{N\left(r, H_{\nu}\right)}<\lambda(\nu=0, \cdots, n-1)$ and $\sum_{\nu=0}^{q-1} \delta\left(H_{\nu}, f\right)=n+1$, then $f$ is of positive integral order, where $\lambda_{N\left(r, I_{\nu}\right)}$ denotes the order of $N\left(r, H_{\nu}\right)$. 
Since $\left\{H_{\nu}\right\}_{\nu=0}^{q-1}$ are in general position, we choose a homogeneous coordinate system $w=\left[w_{0}, \cdots, w_{n}\right]$ on $\boldsymbol{P}^{n}$ so that $H_{\nu}=\left\{w: w_{\nu}=0\right\} \quad(\nu=0, \cdots, n)$. Let $\phi_{\alpha}^{\nu}(w)$ $=\sum_{k} a_{k}^{\nu} w_{k} / w_{\alpha}\left(w_{\alpha} \neq 0\right)$ be the holomorphic section on $L \rightarrow \boldsymbol{P}^{n}$ such that $H_{\nu}=\left\{w: \psi_{\alpha}^{\nu}\right.$ $\left.=0\left(w_{\alpha} \neq 0\right)\right\} \quad(\nu=0, \cdots, q-1)$. Then, for any $p(n+1 \leqq p<\infty)$, we see

$$
\prod_{\nu=0}^{p-1} \frac{\sum_{k=0}^{n}\left|w_{k}\right|^{2}}{\left|\sum_{k=0}^{n} a_{k}^{\nu} w_{k}\right|^{2}} \leqq \kappa \sum_{\left(\begin{array}{c}
p \\
n
\end{array}\right)} \prod_{j=1}^{n}\left\{\frac{\sum_{k=0}^{n}\left|w_{k}\right|^{2}}{\left|\sum_{k=0}^{n} a_{k}^{\nu}, w_{k}\right|^{2}}\right\}
$$

for some constant $\kappa>0$ depending only on $H_{\nu}(\nu=0, \cdots, p)$. We put

$$
\phi_{\nu}(z)=\sum_{k=0}^{n} a_{k}^{\nu} f_{k}(z), \quad \text { so } \phi_{\nu}=f_{\nu} \text { for } \nu=0, \cdots, n \text {. }
$$

We can represent $f$ as $f=\left[f_{0}, \cdots, f_{n}\right]$ so that $f_{2}$ are entire functions of order $\lambda_{\imath}$ and $\lambda_{0}=\lambda_{N\left(r, H_{0}\right)}$. In fact, $f_{0}$ can be represented as

$$
f_{0}(z)=z^{s} e^{G(z)} \prod_{\jmath=1}^{\infty} E\left(\frac{z}{a_{\jmath}}, d\right)
$$

where $E\left(\frac{z}{a,}, d\right)$ is the Weierstrass' primary factor of genus $d$ consisting of the zeros $\left\{a_{j}\right\}$ of $f_{0}, s \in \boldsymbol{Z}$, and $G(z)$ an entire function. We now divide $f_{j}(z)$ by $e^{G(z)}(\jmath=0, \cdots, n)$, and have $\lambda_{0}=\lambda_{N\left(r, H_{0}\right)}$. We also see $\lambda_{\jmath} \leqq \lambda$, since $T_{1}\left(r, f_{j} / f_{\imath}\right)$ $\leqq T(r, f)+O(1)(\imath \neq j)$.

§3. Two lemmas. We need the following lemmas:

Lemma 1. Let $f: \boldsymbol{C} \rightarrow \boldsymbol{P}^{n}$ be a non-degenerate holomorphic curve of finite order and let $\left\{H_{\nu}\right\}_{\nu=0}^{p-1}$ be $p$ hyperplanes $\subset \boldsymbol{P}^{n}$ in general position $(p<\infty)$. Then

$$
\begin{aligned}
\sum_{\nu=0}^{p-1} m\left(r, H_{\nu}\right) \leqq n T(r, f)+ & \frac{1}{2 \pi} \int_{0}^{2 \pi} \log ^{+} \frac{1}{\left|J\left(r e^{i \theta}\right)\right|} d \theta+\sum_{k=0}^{n-1} 2 T_{1}\left(r, f_{k}\right) \\
& +O(\log r)(r \longrightarrow \infty) .
\end{aligned}
$$

Here $f_{k}$ are entire functions defined above and $J \equiv W\left(f_{0}, \cdots, f_{n}\right)$ denotes the Wronskian determinant of $\left\{f_{j}\right\}_{j=0}^{n}$.

Proof. We now estimate $\sum_{\nu=0}^{p-1} m\left(r, H_{\nu}\right)$ by using (1). We see

$$
\begin{aligned}
\sum_{\nu=0}^{p-1} m\left(r, H_{\nu}\right) & =\sum_{\nu=0}^{p-1} \frac{1}{4 \pi} \int_{0}^{2 \pi} \log \frac{\sum_{k=0}^{n}\left|f_{k}\left(r e^{i \theta}\right)\right|^{2}}{\left|\phi_{\nu}\left(r e^{i \theta}\right)\right|^{2}} d \theta \\
& =\frac{1}{4 \pi} \int_{0}^{2 \pi} \log \prod_{\nu=0}^{p-1} \frac{\sum_{k=0}^{n}\left|f_{k}\left(r e^{i \theta}\right)\right|^{2}}{\left|\phi_{\nu}\left(r e^{i \theta}\right)\right|^{2}} d \theta
\end{aligned}
$$




$$
\begin{aligned}
& \leqq \frac{1}{4 \pi} \int_{0}^{2 \pi} \log \kappa\left\{\sum_{\left(\begin{array}{c}
p \\
n
\end{array}\right)} \prod_{j=1}^{n} \frac{\sum_{k=0}^{n}\left|f_{k}\right|^{2}}{\left|\phi_{\nu \jmath}\right|^{2}}\right\} d \theta \\
& =\frac{n}{4 \pi} \int_{0}^{2 \pi} \log \left(\sum_{k=0}^{n}\left|f_{k}\right|^{2}\right) d \theta \\
& +\frac{1}{4 \pi} \int_{0}^{2 \pi} \log \left[\left\{1+\sum_{\left(\begin{array}{l}
p \\
n
\end{array}\right)^{\prime}} \frac{\left|\phi_{0} \cdots \phi_{n-1}\right|^{2}}{\prod_{j=1}^{n}\left|\phi_{\nu}\right|^{2}}\right\} \cdot \frac{1}{\left|\phi_{0} \cdots \phi_{n-1}\right|^{2}}-\right] d \theta \\
& \text { +const. } \quad \Pi \neq\left|\phi_{0} \cdots \phi_{n-1}\right|^{2} \\
& \leqq n T(r, f)+\frac{1}{4 \pi} \int_{0}^{2 \pi} \log +\left\{\sum_{\left(\begin{array}{l}
p \\
n
\end{array}\right)^{\prime}} \frac{\left|\phi_{0} \cdots \phi_{n-1}\right|^{2}}{\prod_{j=1}^{n}\left|\phi_{\nu}\right|^{2}} \cdot \frac{|J|^{2}}{\left|\phi_{0} \cdots \phi_{n-1}\right|^{2}\left(\sum_{k=0}^{n-1}\left|\phi_{k}\right|^{2}\right)}\right\} d \theta \\
& \Pi \neq\left|\phi_{0} \cdots \phi_{n-1}\right|^{2} \\
& +\frac{1}{4 \pi} \int_{0}^{2 \pi} \log ^{+} \frac{\sum_{k=0}^{n-1}\left|\phi_{k}\right|^{2}}{|J|^{2}} d \theta+\frac{1}{2 \pi} \int_{0}^{2 \pi} \log ^{+} \frac{1}{\left|\phi_{0} \cdots \phi_{n-1}\right|} d \theta+\text { const . } \\
& \leqq n T(r, f)+\frac{1}{4 \pi} \int_{0}^{2 \pi} \log ^{+} \sum_{\left(\begin{array}{l}
p \\
n
\end{array}\right)^{\prime}} \frac{|J|^{2}}{\prod_{j=1}^{n}\left|\phi_{\nu_{\jmath}}\right|^{2}\left(\sum_{k=0}^{n-1}\left|\phi_{k}\right|^{2}\right)} d \theta \\
& \Pi \neq\left|\phi_{0} \cdots \phi_{n-1}\right|^{2} \\
& +\frac{1}{2 \pi} \int_{0}^{2 \pi} \log ^{+} \frac{1}{|J|} d \theta+\sum_{k=0}^{n-1} \frac{1}{2 \pi} \int_{0}^{2 \pi} \log ^{+}\left|\phi_{k}\right| d \theta \\
& +\sum_{k=0}^{n-1} \frac{1}{2 \pi} \int_{0}^{2 \pi} \log ^{+} \frac{1}{\left|\phi_{k}\right|} d \theta+\text { const } . \\
& \leqq n T(r, f)+\frac{1}{2 \pi} \int_{0}^{2 \pi} \log ^{+} \frac{1}{|J|} d \theta+2 \sum_{k=0}^{n-1} T_{1}\left(r, f_{k}\right)+O(\log r) \quad(r \longrightarrow \infty),
\end{aligned}
$$

since $\left\{H_{\nu}\right\}_{\nu=0}^{p-1}$ are in general position, $\log ^{+}|a \cdot b| \leqq \log ^{+}|a|+\log ^{+}|b|, \log ^{+}|a+b|$ $\leqq \log ^{+}|a|+\log ^{+}|b|+\log 2$ and $\frac{1}{2 \pi} \int_{0}^{2 \pi} \log ^{+}\left|\phi_{\nu}^{\prime}\right| /\left|\phi_{\nu}\right| d \theta=O(\log r)$ for a meromorphic function $\phi_{\nu}$ of finite order. Here $\sum_{\left(\begin{array}{c}p \\ n\end{array}\right)^{\prime}}$ means $\sum_{\left(\begin{array}{l}p \\ n\end{array}\right)}$ deleting the term of $\mid \phi_{0} \cdots$ $\left.\phi_{n-1}\right|^{2}$. This proves Lemma 1 .

LEMMA 2 (Mori [3], p. 666-667). Let $f$, be $n+1$ linearly independent meromorphic functions of order $\lambda_{\jmath}$ (in the sense of order of $\left.T_{1}\left(r, f_{j}\right)\right)(\jmath=0, \cdots, n)$. Put $J=W\left(f_{0}, \cdots, f_{n}\right)$ be the Wronskian determinant of $\left\{f_{j}\right\}$. If $\lambda_{j}<\lambda_{n}$ for $j=0$, $\cdots, n-1$, then $J$ has order $\lambda_{n}$. 
$\S 4$. Proof of Theorem. If $f$ has order $\lambda$ which is not an integer, then $f_{j}$ has order $<\lambda(\jmath=0, \cdots, n-1)$. For, if there exists an $f_{\jmath}(0 \leqq j \leqq n-1)$ with $\lambda_{j}=\lambda$, then $\lambda=\lambda_{j}$ is an integer, since $N_{1}\left(r, 1 / f_{j}\right)=N\left(r, H_{j}\right)$ is of order $\left\langle\lambda=\lambda_{j}\right.$. This is a contradiction. Hence $f_{n}$ has order $\lambda_{n}=\lambda$.

We also see that $J$ is of order $\lambda$. For, $f=\left[f_{0}, \cdots, f_{n}\right]$ repesented above is a non-degenerate holomorphic curve, so $f$, are linearly independent entire functions of order $\lambda_{j}<\lambda(\jmath=0, \cdots, n-1)$ and $\lambda_{n}=\lambda$. Hence, by Lemma $2, J$ is of order $\lambda_{n}=\lambda$.

By the assumption $\sum_{\nu=0}^{q} \delta\left(H_{\nu}, f\right)=n+1$, for any $\eta>0$ there exists an integer $p=p(\eta)$ such that

$$
\sum_{\nu=0}^{n-1} \delta\left(H_{\nu}, f\right) \geqq n+1-\frac{\eta}{2} .
$$

By definition of $\delta\left(H_{\nu}, f\right)=\liminf _{r \rightarrow \infty}\left\{m\left(r, H_{\nu}\right) / T(r, f)\right\}$, for any $\varepsilon_{\nu}>0$ there is an $r_{\varepsilon_{\nu}}>0$ such that

$$
m\left(r, H_{\nu}\right)>\left(\delta\left(H_{\nu}, f\right)-\varepsilon_{\nu}\right) \cdot T(r, f),
$$

if $r \geqq r_{\varepsilon, r}$. We now choose $\varepsilon_{\nu}$ so small that $\varepsilon=\sum_{\nu=0}^{p-1} \varepsilon_{\nu}<\frac{\eta}{2}$. Then there is an $r_{\varepsilon, \eta}$ such that

$$
\sum_{\nu=0}^{p-1} m\left(r, H_{\nu}\right) \geqq(n+1-\eta) \cdot T(r, f)
$$

for all $r \geqq r_{\varepsilon, r}$. Thus, by (2), we have

$$
\begin{aligned}
(1-o(1)-\eta) \cdot T(r, f) \leqq & \frac{1}{2 \pi} \int_{0}^{2 \pi} \log ^{+} \frac{1}{|J|} d \theta \\
& +2 \sum_{k=0}^{n-1} T_{1}\left(r, f_{k}\right)+O(\log r), \quad(r \longrightarrow \infty) .
\end{aligned}
$$

We note that

$$
\begin{aligned}
T_{1}(r, J) & =\frac{1}{2 \pi} \int_{0}^{2 \pi} \log ^{+}|J| d \theta \leqq \sum_{k=0}^{n} T_{1}\left(r, f_{k}\right)+O(\log r) \\
& \leqq \sum_{k=0}^{n-1} T_{1}\left(r, f_{k}\right)+T_{1}\left(r, f_{n} / f_{0}\right)+T_{1}\left(r, f_{0}\right)+O(\log r) \\
& \leqq T(r, f)+\sum_{k=0}^{n-1} T_{1}\left(r, f_{k}\right)+T_{1}\left(r, f_{0}\right)+O(\log r),(r \longrightarrow \infty)
\end{aligned}
$$

We obtain from (3) and (4),

$$
1-\eta=\liminf _{r \rightarrow \infty} \frac{(1-o(1)-\eta) T(r, f)}{(1+o(1)) T(r, f)} \leqq \liminf _{r \rightarrow \infty} \frac{(1-o(1)-\eta) T(r, f)}{T_{1}(r, J)-\sum_{k=0}^{n-1} 3 T_{1}\left(r, f_{k}\right)}
$$




$$
\leqq \liminf _{r \rightarrow \infty}\left\{\frac{m_{1}(r, 1 / J)+\sum_{k=0}^{n-1} T_{1}\left(r, f_{k}\right)}{T_{1}(r, J)-\sum_{k=0}^{n-1} 3 T_{1}\left(r, f_{k}\right)}\right\} .
$$

Hence, for a sequence $\left\{r_{n}\right\}_{n=1}^{\infty}$ of $\lim _{r_{n} \rightarrow \infty}\left(\log T_{1}\left(r_{n}, J\right) / \log r_{n}\right)=\lambda$, we obtain

$$
1-\eta \leqq \lim _{r_{n} \rightarrow \infty} \frac{m_{1}\left(r_{n}, 1 / J\right)}{T_{1}\left(r_{n}, J\right)}
$$

such a sequence exists since $J$ has order $\lambda$. On the other hand, we see $T_{1}(r, J)$ $=m_{1}(r, J)$, since $J$ is an entire function. Thus we have

$$
\lim _{r_{n} \rightarrow \infty} \frac{m_{1}\left(r_{n}, J\right)+m_{1}\left(r_{n}, 1 / J\right)}{T_{1}\left(r_{n}, J\right)} \geqq 2-\eta .
$$

Since we can choose Pólya peaks $\left\{r_{n}\right\}$ of $T(r, f)$ such that

$$
\lim _{r_{n} \rightarrow \infty}\left(\log T_{1}\left(r_{n}, J\right) / \log r_{n}\right)=\lambda,
$$

we obtain

$$
\lim _{r_{n} \rightarrow \infty} \frac{m_{1}\left(r_{n}, J\right)+m_{1}\left(r_{n}, 1 / J\right)}{T_{1}\left(r_{n}, J\right)} \leqq \chi(\lambda)
$$

where

$$
\chi(\lambda)<2-\frac{([\lambda]+1-\lambda)(\lambda-[\lambda])}{2 \lambda([\lambda]+1)(2+\log ([\lambda]+1))} \quad \text { if } \quad \lambda>1
$$

and

$$
\chi(\lambda)<1+\lambda \quad \text { if } \lambda<1
$$

by a proof of Nevanlinna's theorem ([2], p. 101-103). Hence we obtain a contradiction if we take $\eta$ sufficiently small. Therefore we have $\lambda \in \boldsymbol{Z}^{+}$. Here $\lambda>0$ is a consequence from [4] or [7], since there are $n+1$ hyperplanes $\left\{H_{2}\right\}$ in general position with $\delta\left(H_{\nu}, f\right)>0$. This completes the proof of Theorem.

Remark 1. We can construct a holomorphic curve $f$ which satisfies the conditions of Theorem and has more than $n+1$ deficient hyperplanes. Here the deficient hyperplane means the hyperplane $H$ with $\delta(H, f)>0$.

Let $w=\left[w_{0}, w_{1}, w_{2}\right]$ be a homogeneous coordinate system in $\boldsymbol{P}^{2}$. Consider $f: \boldsymbol{C} \rightarrow \boldsymbol{P}^{2}$ a non-degenerate holomorphic curve given by $f=\left[1, e^{2 z}, e^{2 z}-e^{z}\right]$, and hyperplanes $H_{\nu}=\left\{w: w_{\nu}=0\right\},(\nu=0,1,2)$ and $H_{3}=\left\{w: w_{0}-w_{1}+w_{2}=0\right\}$. Then we see that $T(r, f)=\frac{2 r}{\pi}+O(1), N\left(r, H_{\nu}\right)=0(\nu=0,1)$ and $N\left(r, H_{\nu}\right)=\frac{r}{\pi}+O(\log r)$ $(\nu=2,3)$. Hence we obtain that $\delta\left(H_{0}, f\right)=\delta\left(H_{1}, f\right)=1, \delta\left(H_{2}, f\right)=\delta\left(H_{3}, f\right)==\frac{1}{2}$ and $f$ is of order one.

Remark 2. Prof. Toda told me that the conditions $\lambda_{N\left(H_{j}, f\right)}<\lambda$ and $\sum_{\nu=0}^{q} \delta\left(H_{\nu}, f\right)$ 
$=n+1$ implies $\delta\left(H_{\jmath}, f\right)=1$ by using the theory of his modified deficiency. (See Toda [8]).

\section{REFERENCES}

[1] EdRei, A. AND W. H.J. Fuchs, On the growth of meromorphic functions with several deficient values, Trans. Amer. Math. Soc. 93 (1959), 293-328.

[2] Hayman, W.K., Meromorphic functions, Oxford Math. Mono., (1964).

[3] MORI, S., Sum of deficiencies and the order of a meromorphic function, Tôhoku Math. J., Vol. 22 (1970), 659-669.

[4] Mori, S., On the deficiencies of meromorphic mappings of $C^{n}$ into $P^{N} C$, Nagoya Math. J., 67 (1977). 165-176.

[5] Noguchi, J., A relation between order and defect of meromorphic mapping of $C^{n}$ into $P^{N} C$, Nagoya Math. J., Vol. 59 (1975), 97-106.

[6] SToll, W., Die beiden Hauptsätze Wertverteilungstheorie bei Funktionen mehrerer komplexen Veränderlichen (I) (II), Acta Math., 90 (1953), 1-115 and 92 (1954), 55-169.

[7. TODA, N., Sur la croissance de fonctions algebroides á valeurs deficientes, Kōda1 Math. Sem. Rep., 22 (1970), 324-337.

[8] TODA, N., On a modified deficiency of meromorphic functions, Tôhoku Math. J., Vol. 22 (1970), 635-658.

[9] VitTer, A., The lemma of the logarithmic derivative in several complex varıables, Duke Math. J., Vol. 44 (1977), 89-104.

Mathematical Institute

TÔHOKU UNIVERSITY

Sendai, JAPAN 